\title{
Immune context characterization and heterogeneity in primary tumors and pulmonary metastases from renal cell carcinoma
}

\author{
Melissa Bersanelli*,‡,1, Letizia Gnetti ${ }^{\ddagger 2}$, Elena Varotti², Luca Ampollini ${ }^{3}$, Paolo \\ Carbognani $^{3}$, Francesco Leonardi ${ }^{1}$, Michele Rusca ${ }^{3}$, Nicoletta Campanini ${ }^{2}$, Francesco \\ Ziglioli ${ }^{4}$, Clara I Dadomo ${ }^{2}$, Francesco P Pilato ${ }^{2}$, Alessio Cortellini $^{5}$, Elena Rapacchi ${ }^{1}$, \\ Giuseppe Caruso ${ }^{1}$, Enrico M Silini ${ }^{2}$, Umberto Maestroni ${ }^{4}$ \& Sebastiano Buti ${ }^{1}$ \\ ${ }^{1}$ Department of Medical Oncology, University Hospital of Parma, Parma, 43126, Italy \\ ${ }^{2}$ Department of Pathological Anatomy \& Histology, University Hospital of Parma, Parma, 43126, Italy \\ ${ }^{3}$ Department of Thoracic Surgery, University Hospital of Parma, Parma, 43126, Italy \\ ${ }^{4}$ Department of Urology, University Hospital of Parma, Parma, 43126, Italy \\ ${ }^{5}$ Department of Biotechnological \& Applied Clinical Sciences, Medical Oncology, St Salvatore Hospital, University of L'Aquila, \\ L'Aquila, 67100, Italy \\ *Author for correspondence: Tel..: +39 0521702 316; bersamel@libero.it \\ ${ }^{\ddagger}$ Authors contributed equally
}

\begin{abstract}
Aim: The knowledge of the immune context of renal cell carcinoma (RCC) is useful to predict benefit from immunotherapy. We retrospectively characterized the immune context of RCC patients underwent primary nephrectomy and pulmonary metastasectomy. Materials \& methods: Intratumoral infiltrating lymphocytes and peritumoral renal infiltrating lymphocytes, lymphocyte subpopulations (CD4+ $\left.{ }^{+}, \mathrm{CD}^{+}\right)$, PD-1, PD-L1 were explored in paired samples of primary RCC ( $T$ ) and respective pulmonary metastases (M). Results: The immune variables demonstrated intralesional and intratumoral heterogeneity. Intralesional lymphocyte heterogeneity reached $76 \%$ of cases in T, $28 \%$ in M. The heterogeneity rate for PD-L1 expression was from $44 \%$ (T) to $56 \%$ (M); it correlated with better survival. Conclusion: The immune context of RCC is highly variable both within a given tumor and among primary and metastases.
\end{abstract}

First draft submitted: 15 July 2018; Accepted for publication: 11 October 2018; Published online: 7 December 2018

Keywords: CD4 • CD8 • heterogeneity • immune context • lymphocyte subpopulations • PD-1 • PD-L1 • renal cell carcinoma $\bullet$ RILS $\bullet$ TILS

Renal cell carcinoma (RCC) has been defined as an immunogenic tumor with a prominent dysfunctional immune cell infiltrate, unable to control the tumor growth [1]. The investigational interest for the immune context of RCC has recently increased, due to the therapeutic use of immune checkpoint inhibitors (CKIs) in clinical practice [2]. The anti-PD-1 nivolumab was recently approved for metastatic RCC (mRCC) patients pretreated with VEGF receptor (VEGFR) inhibitors [3]. In addition, its combination with the CTLA-4 inhibitor ipilimumab demonstrated superiority over the first-line standard anti-VEGF (VEGFR) sunitinib to improve the outcome of untreated mRCC patients with intermediate- or poor-risk features according to the International Metastatic Renal Cell Carcinoma Database Consortium model [4].

In RCC, PD-1 is expressed in the lymphoid cells infiltrating the tumor and its stroma. The levels of PD-1 expression in intratumoral-infiltrating lymphocytes (TILs) and peritumoral renal-infiltrating lymphocytes (RILs) correlate with advanced stage, grade and prognosis. Its ligand PD-L1 expression in RCC tumor cells is also associated with worse clinical outcomes $[5,6]$.

Whether in several tumors models the presence of infiltrating lymphocytes correlates with a good prognosis, as expression of a tissue immune response [7], in RCC this relationship is reversed. Several authors reported an unfavorable prognostic role of TILs levels showing an association with high tumor grade and stage [8,9]. There are

Future Medicine 
still conflicting evidences, however, about the T-cell phenotype potentially related to the worse prognosis, whether $\mathrm{CD}^{+}$or $\mathrm{CD}^{+}$lymphocytes [9-10]. A role for Treg has also been suggested [11,12].

Few studies have so-far addressed the issue of PD-L1 expression as assessed by immunohistochemistry (IHC) in RCC, showing an association between PD-L1 overexpression and shorter survival [13-16]. In clinical trials of CKIs therapy, the predictive value of PD-L1 expression on tumor cells remains ambiguous: although it may increase the likelihood of benefit with anti-PD-1, it failed to identify all responders [17]. Moreover, the lack of agreement about the cutoff value of expression is still a major issue $[18,19]$. Conversely, PD-L1 expression on the immune cells infiltrating the tumor showed a positive correlation with disease-free survival, suggesting different ways to assess such biomarker in future studies [20]. Resistance to treatment with nivolumab due to the lack of expression of PD-1 has also been suggested [5]. Finally, a negative predictive value of PD-L1 expression in tumor cells for treatment with tyrosine-kinase inhibitors was reported, but still requires validation [21].

Deep-sequencing analyses of solid tumors have identified extensive intra- and inter-tumor genetic heterogeneity. Intratumor genetic heterogeneity (ITH) can contribute to treatment failure and drug resistance and it has major implications for personalized medicine approaches that rely on single-biopsy samples to portray tumor mutational profiles. Clear-cell RCC (ccRCC) is a well-studied model for genetic tumor heterogeneity, with increasing ITH according to the number of biopsies analyzed without evidence of saturation [22]. In ccRCC, ITH segregates according to a pattern of branched clonal diversification that correlates with the acquisition of distinct driver mutations [23]. .

In parallel, a recent retrospective study identified major variation of clinical responses to antiangiogenic tyrosinekinase inhibitor among metastases of individual RCC patients, suggesting that genetic heterogeneity upon tumor progression underlies variability of clinical phenotypes [24]. This clinical phenomenon mirrors molecular ITH. According to these results, antiangiogenic drug resistance was a feature confined to subclones and rooted in the tumors' phylogenetic tree. Clinical progression was seen for new rather than existing metastases, supporting a selective pressure on disease phenotype.

The issue of heterogeneity also concerns the expression of immune biomarkers in RCC. Jilaveanu et al. first evidenced a weak correlation between PD-L1 expression in primary and metastatic sites for a given patient and suggested that PD-L1 staining in nephrectomy specimens cannot be used to select mRCC patients for CKI therapy. Moreover, they demonstrated that variable PD-L1 staining heterogeneity is present within individual tumors and it is maintained in metastases [25]. Similar results were subsequently reported by Callea et al., hypothesizing that PD-L1 levels assessed in random samples of the primary tumors may not accurately reflect expression levels in metastatic lesions, which are the main target of systemic therapy. In their case series, discordant PD-L1 staining between primary tumors and metastases was observed in more than $20 \%$ of cases, a figure that suggests that PD-L1 assessment should be performed in the secondary lesions [26]. Furthermore, in a recent report, PD-L1 expression was higher in RCC with T effector gene expression signature, and it was also related to more aggressive histology (sarcomatoid RCC), suggesting that genetic heterogeneity and the immune context can be closely linked to each other [27].

The aim of this explorative study was the characterization of the immune context and of its intralesional and intratumoral heterogeneity, using easily accessible laboratory techniques, in mRCC patients who underwent primary nephrectomy and resection of synchronous or metachronous pulmonary metastases. TILs and RILs, T-lymphocyte subpopulations $\left(\mathrm{CD}^{+}\right.$and $\left.\mathrm{CD}^{+}\right)$and expression of PD-1 and PD-L1 were assessed by immunostaining in different tumor areas both in primary and secondary lesions. The mutual relation between the different immune variables was also evaluated as well as their correlation with the patients' outcome.

\section{Materials \& methods}

This was a monocentric, retrospective, explorative study, approved by the Local Ethical Committee of Parma. We enrolled all consecutive RCC patients with partial or radical nephrectomy performed from September 1998 to October 2014 and subsequently underwent resection for synchronous or metachronous pulmonary metastases at our Thoracic Surgery Unit. The inclusion criteria were: availability of tumor tissue from both primary tumor and metastasis, a volume of tissue adequate to perform all planned analyses and availability of complete clinical records. The study procedure included complete histopathological revision of available slides from primary and metastasis; then, on both paired samples, we performed: qualitative and quantitative assessment of TILs and RILs, quantitative analysis of $\mathrm{CD}^{+}$and $\mathrm{CD} 4^{+}$lymphocyte subpopulations in TILs and RILs, qualitative and quantitative assessment of PD-L1 on TILs, RILs and tumor cells; qualitative and quantitative assessment of PD-1 on TILs and RILs. The 
immunohistochemical expression of the different biomarkers was evaluated by independent review of two experts. Discordant cases, or cases with not homogeneous staining, due to necrosis or poor conservation, were revised in consensus and those lacking valid internal control were excluded and reexamined on a new sample. For each sample, absolute percentage of positive cells and staining intensity scored from 0 to $1+, 2++, 3+++$ were recorded.

Intralesional and intratumor heterogeneity of CD4, CD8 and PD-L1 stains were defined as follows.

- The absolute number of $\mathrm{CD} 4^{+}$and $\mathrm{CD} 8^{+}$lymphocytes was counted in four different high-power fields (HPF) at 40x magnification from different sections of each compartment (TILs and RILs) of both primary and metastasis; the mean value of the four counts was assigned as the total amount of $\mathrm{CD}^{+} \mathrm{CD} 8^{+}$cells. The coefficient of variation $(\mathrm{V})$, defined as the ratio of the standard deviation $(\sigma)$ to the mean, was calculated to quantify heterogeneity as the extent of variability in relation to the mean value. Cases with $V \geq 0.5$ (coefficient of variation of at least 50\%) were considered as heterogeneous, while cases with $V<0.5$ (variation inferior to $50 \%$ ) were defined as homogeneous (the more $V$ was near to 0 , the more the mean value was considered as reliable) $[28,29]$.

- The percentage of PD-L1-positive cells (membranous expression) was calculated in four different HPF from different sections of each compartment (tumor cells, TILs and RILs) of both primary and metastasis. Data were analyzed as a continuous variable and also transformed in a categorical binary variable (positive vs negative; qualitative measurement) using a cutoff value of at least $1 \%$ positive cells to define a case as PD-L1 positive. Intralesional PD-L1 heterogeneity was defined for at least one discordant qualitative measurement from the four HPF 40x; the same procedure was repeated for each pulmonary metastasis.

- Data were compared from primary tumor and metastasis to assess intratumor heteronegeity.

Detailed laboratory procedures are reported in the Supplementary materials section.

The statistical analysis included $t$ test for parametric data and Pearson's $\chi^{2}$ test or Fisher test for nonparametric data. Kaplan-Meier curves were used to estimate the survival function and the log-rank test was calculated to compare the survival distributions according to the different variables. Surgical interval was defined as the time interval between nephrectomy and metastasectomy. Overall survival (OS) from nephrectomy and from metastasectomy were measured as the time interval from surgical intervention to death for any reason, or to the last follow-up for alive patients.

\section{Results}

\section{Study population}

Overall, 25 patients were included in the study: 10 were females and 15 were males; their median age was 65 years (range 55 to 78). The main clinical and pathological features of the series are summarized in Table 1. The histologic subtype of the primary tumor was ccRCC for 24 patients; a single patient had a mixed histology, with clear cell and papillary components (Supplementary Figure 1). Pulmonary metastases were synchronous in 4 cases and metachronous in 21 cases; the onset of a resected pulmonary metastases did not always represent the first disease recurrence after nephrectomy. The surgical interval between nephrectomy and metastasectomy was 32.6 months (95\% CI: 15.7-49.4, no censored cases; mean of 35.4 months). At a median follow-up of 9.8 years (95\% CI: 8.7-10.9), the median OS (mOS; as assessed from nephrectomy) was of 8.1 years (95\% CI: 3.7-12.5), with 13 $(52 \%)$ censored cases (Supplementary Figure 2). The mOS from pulmonary metastasectomy was of 5.5 years (95\% CI: 1.9-9.1), with 13 censored cases (52\%) at the median follow-up of 7.1 years (95\% CI: 4.04-10.16) metastasectomy (Supplementary Figure 3).

\section{The immune context}

The immune context was explored by the analysis of TILs and perilesional RILs, $\mathrm{CD} 8^{+}$and $\mathrm{CD} 4^{+}$subpopulations and expression of PD-1 and PD-L1 in different cell compartments. Table 2 summarizes the results of the qualitative evaluation of TILs and RILs and their characterizations as to $\mathrm{CD} 4^{+}$and $\mathrm{CD} 8^{+} \mathrm{T}$-cells ratios (as shown in Figure 1). TILs and RILs were detectable in most cases, either in primary tumor or metastasis, with variable density (Figure 2). Overall, TILs and RILs scores were, respectively, increased in 36\%, unchanged in $44 \%$, decreased in $20 \%$ of cases, from the primary to the secondary lesion (Table 2).

PD-L1 was expressed on tumor cells in $24 \%$ of cases in the primary tumor and in $24 \%$ of cases in the metastases, with low mean intensity (see Table 3 for quantitative details). PD-L1 expression in the immune infiltrate was more 
Table 1. Clinical characteristics of the study population and histopathological features of the analyzed samples from nephrectomies and pulmonary resections.

\begin{tabular}{|c|c|c|c|c|c|}
\hline \multicolumn{2}{|c|}{$\begin{array}{l}\text { Clinical characteristics (Median age } 65 \text { years [range } \\
\qquad 55-78] \text { ) }\end{array}$} & \multicolumn{2}{|c|}{ Primary tumor features } & \multicolumn{2}{|c|}{ Pulmonary metastasis features } \\
\hline & No. of patients (\%) & & No. of patients (\%) & & No. of patients $(\%)^{*}$ \\
\hline Gender & & T size & & No. of metastases & \\
\hline M & $15(60)$ & $\leq 4 \mathrm{~cm}$ & $3(12)$ & Single & $12(48)$ \\
\hline \multirow[t]{3}{*}{$\mathrm{F}$} & $10(40)$ & $>4 \mathrm{~cm}$ but $\leq 7 \mathrm{~cm}$ & $12(48)$ & Multiple & $13(52)$ \\
\hline & & $>7 \mathrm{~cm}$ but $\leq 10 \mathrm{~cm}$ & $6(24)$ & & \\
\hline & & $>10 \mathrm{~cm}$ & $4(16)$ & & \\
\hline Stage at diagnosis & & Histotype & & Histotype & \\
\hline 1 & $11(44)$ & Clear cell & $24(96)$ & Clear cell & $23(92)$ \\
\hline II & $6(24)$ & Sarcomatoid & $0(0)$ & Sarcomatoid & $1(4)$ \\
\hline III & $4(16)$ & Others & $1(4)$ & Others & $1(4)$ \\
\hline IV & $4(16)$ & & & & \\
\hline \multicolumn{2}{|l|}{ Type of nephrectomy } & Fuhrman grade & & Laterality & \\
\hline Partial & $1(4)$ & G1 & $0(0)$ & Left lung & $7(28)$ \\
\hline Radical & $24(96)$ & $\mathrm{G} 2$ & $5(20)$ & Right lung & $14(56)$ \\
\hline With lymphadenectomy & $1(4)$ & G3 & $14(56)$ & Bilateral involvement & $4(16)$ \\
\hline With adrenalectomy & $4(16)$ & G4 & $6(24)$ & & \\
\hline \multicolumn{2}{|l|}{ Intent of nephrectomy } & \multicolumn{2}{|l|}{ Vascular invasion } & \multicolumn{2}{|l|}{ Nodal involvement } \\
\hline Palliative (cytoreduction) & $4(16)$ & Yes & $15(60)$ & Yes & $3(12)$ \\
\hline \multirow[t]{2}{*}{ Curative } & $21(84)$ & No & $10(40)$ & No & $3(12)$ \\
\hline & & & & Unknown (*not resected) & $21(84)$ \\
\hline \multirow{2}{*}{\multicolumn{2}{|c|}{$\begin{array}{l}\text { Systemic treatment after } \\
\text { nephrectomy }\end{array}$}} & Necrosis & & Necrosis & \\
\hline & & 0 & $9(36)$ & 0 & $17(68)$ \\
\hline Any & $20(80)$ & $1(+)$ & $5(20)$ & $1(+)$ & $3(12)$ \\
\hline Adjuvant & $3(12)$ & $2(++)$ & $7(28)$ & $2(++)$ & $2(8)$ \\
\hline I line & $2(8)$ & $3(+++)$ & $4(16)$ & $3(+++)$ & $3(12)$ \\
\hline
\end{tabular}

\section{Table 2. Characterization of the immune context of primary tumors and pulmonary metastases.}

\begin{tabular}{|llll|}
\hline Primary tumor & No. of patients (\%) & Pulmonary metastasis & No. of patients (\%) \\
\hline TILs & & TlLs & $1(4)$ \\
0 & $1(4)$ & 0 & $11(44)$ \\
$1(+)$ & $13(52)$ & $1(+)$ & $10(40)$ \\
$2(++)$ & $9(36)$ & $2(++)$ & $3(12)$ \\
$3(+++)$ & $2(8)$ & $3(+++)$ & $2(8)$ \\
\hline RILs & & RILs & $9(36)$ \\
0 & $0(0)$ & 0 & $13(52)$ \\
$1(+)$ & $16(64)$ & $1(+)$ & $1(4)$ \\
$2(++)$ & $7(28)$ & $2(++)$ & $6(24)$ \\
$3(+++)$ & $2(8)$ & $3(+++)$ & $8(32)$ \\
\hline CD4/CD8 ratio TILs & & CD4/CD8 ratio TILs & $11(44)$ \\
$\leq 1$ & $12(48)$ & $\leq 1$ & $4(16)$ \\
$>1$ but $\leq 2$ & $5(20)$ & $>1$ but $\leq 2$ & $9(36)$ \\
$>2$ & $8(32)$ & $>2$ & $12(48)$ \\
\hline CD4/CD8 ratio RILs & CD4/CD8 ratio RILs & \\
$\leq 1$ & & $\leq 1$ & \\
$>1$ but $\leq 2$ & $4(16)$ & $>1$ but $\leq 2$ & \\
$>2$ & $3(12)$ & $>2$ & \\
\hline RIL: Renal-infiltrating lymphocyte; TIL: Tumor-infiltrating lymphocyte. & & \\
\hline
\end{tabular}

frequent, in both primaries, 36\% in TILs and 32\% in RILs, and metastases, 52\% in TILs and 60\% in RILs.

PD-1 was expressed in $68 \%$ of primary tumors on RILs and in $72 \%$ of cases on RILs; it was also frequently expressed in the metastases, $64 \%$ of cases on TILs and $80 \%$ of cases on RILs (see Table 3 for quantitative details).

Intratumoral \& intralesional heterogeneity

The analysis of intralesional heterogeneity (within each lesion, either primary or metastasis) for CD4 ${ }^{+}$and $\mathrm{CD}^{+}$ lymphocytes subpopulations evidenced high rates of variation. The highest variation was for TILs of the primary tumor: $48 \%$ of cases were heterogeneous for $\mathrm{CD}^{+}$expression and $36 \%$ for $\mathrm{CD}^{+}$; overall, $52 \%$ of cases were heterogeneous for at least one variable (Supplementary Table 2). As to RILs of the primary tumor, 24\% of cases 


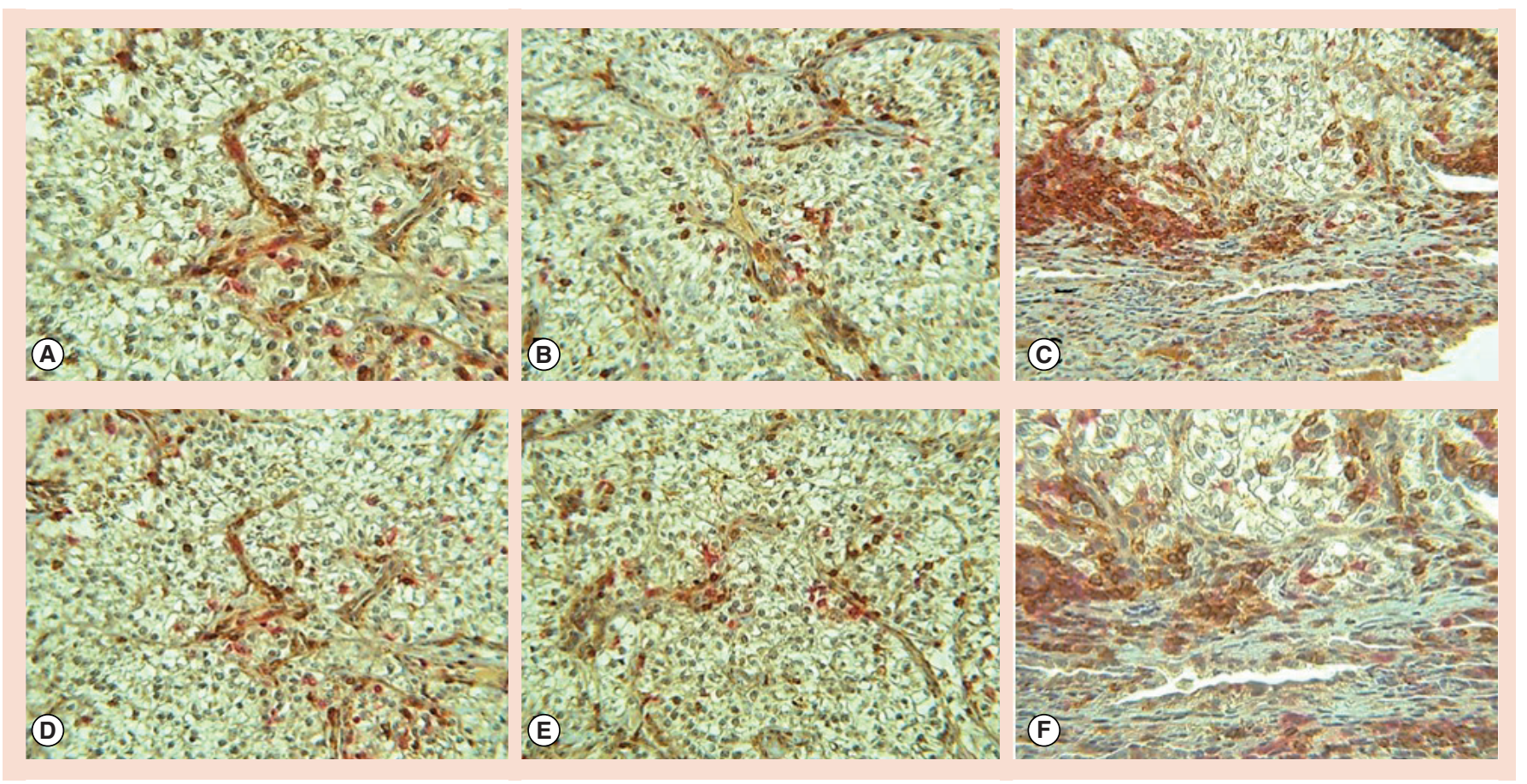

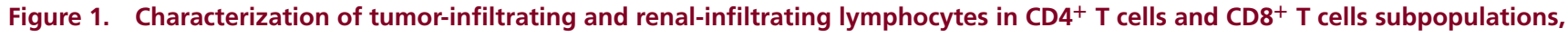
assessed by immunohistochemistry. (A, B, D \& E) IHC for CD4/CD8 in TILs from primary renal cell carcinoma (40x); (C \& F) IHC for CD4/CD8 in RILs from primary renal cell carcinoma (20x and 40x, respectively).

IHC: Immunohistochemistry; RIL: Renal-infiltrating lymphocyte; TIL: Tumor-infiltrating lymphocyte.

\begin{tabular}{|c|c|c|c|c|c|c|}
\hline \multirow[t]{2}{*}{ Biomarker } & \multicolumn{3}{|c|}{ Primary tumor } & \multicolumn{3}{|c|}{ Pulmonary metastasis } \\
\hline & Tumor cells & TILs & RILs & Tumor cells & TILs & RILs \\
\hline+ & 5 & 7 & 7 & 3 & 11 & 12 \\
\hline++ & 0 & 2 & 0 & 0 & 2 & 3 \\
\hline+++ & 1 & 0 & 0 & 3 & 1 & 1 \\
\hline \multicolumn{4}{|c|}{ PD-1 (\% positive cells) } & - & & \\
\hline Mean & & 26.4 & 24.3 & & 18.2 & 24.9 \\
\hline Median & - & 20 & 20 & & 1 & 10 \\
\hline Range & & $0-80$ & $0-80$ & & $0-80$ & $0-80$ \\
\hline
\end{tabular}

RIL: Renal-infiltrating lymphocyte; TIL: Tumor-infiltrating lymphocyte.

were heterogeneous for $\mathrm{CD}_{4}^{+}$cells and $68 \%$ for $\mathrm{CD}^{+}$; overall, $76 \%$ of cases were heterogeneous for at least one variable (Supplementary Table 3). High rates of CD4 and CD8 heterogeneity were also found in the metastases (Supplementary Tables 4 \& 5): $24 \%$ of cases were heterogeneous for $\mathrm{CD}^{+}$and $28 \%$ for $\mathrm{CD} 8^{+}$in TILs (overall, $28 \%$ of cases were heterogeneous for at least one variable); $36 \%$ of cases were heterogeneous for $\mathrm{CD}^{+}{ }^{+}$and $36 \%$ for $\mathrm{CD}^{+}$in RILs (overall, $48 \%$ of cases were heterogeneous for at least one variable).

The analysis of intratumoral heterogeneity, on the contrary, did not evidenced significant variations between the mean number of $\mathrm{CD}^{+}$or $\mathrm{CD}^{+}$lymphocytes from the primary tumor to the metastasis, nor in TILs or in RILs.

PD-L1 expression showed significant variation of positive cells percentage among different fields of the same tumor. Samples, however, were considered heterogeneous only when showing at least one qualitatively discordant field (namely, positive vs negative fields for PD-L1; a heterogeneous case is shown in Figure 3). Based on this 

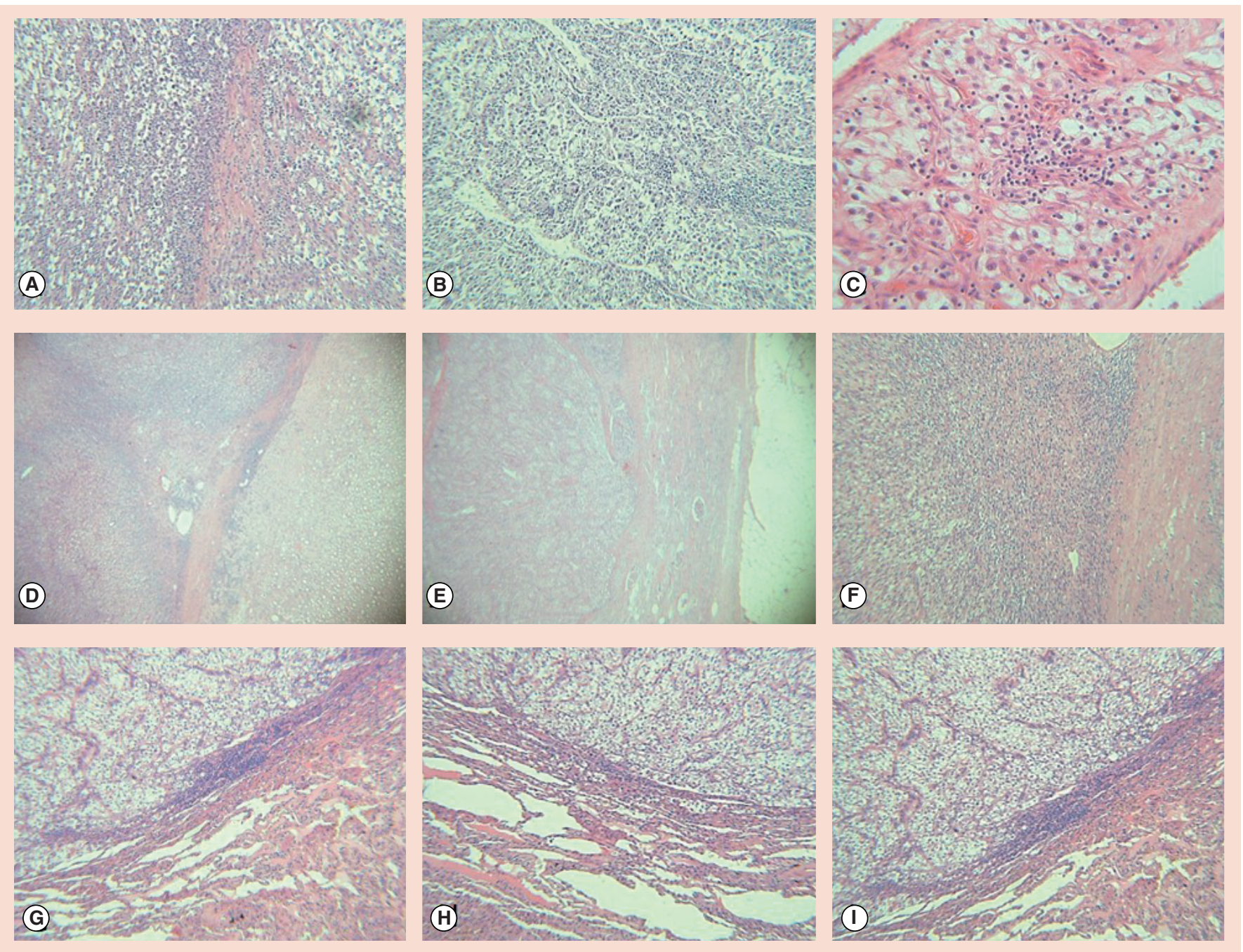

Figure 2. Tumor-infiltrating lymphocytes and peritumoral renal-infiltrating lymphocytes in samples from the study population. (A-C) Presence of intratumoral TILs in three cases of renal cell carcinoma, primary tumor (respectively, 20x, 20x, 40x). (D-F) Presence of peritumoral RILs in three cases of renal cell carcinoma, primary tumor (respectively, 4x, 10x, 20x). (G-I) Presence of RILs in three cases of pulmonary metastases from renal cell carcinoma (all 4x).

RIL: Renal-infiltrating lymphocyte; TIL: Tumor-infiltrating lymphocyte.

criterion, intralesional heterogeneity for PD-L1 expression was found in $20 \%$ of cases on tumor cells, $24 \%$ on TILs and $28 \%$ on RILs (overall, $44 \%$ of cases were heterogeneous in at least one cell compartment; Supplementary Table 6). Intralesional PD-L1 heterogeneity was even higher in metastases: $16 \%$ of metastasis were heterogeneous for PD-L1 expression on tumor cells, $36 \%$ on TILs and $32 \%$ on RILs (overall, $56 \%$ of cases were heterogeneous in at least one cell compartment; Supplementary Table 7). Significant discrepancies in PD-L1 expression were also observed between paired cell compartments from primary tumor and metastasis of the same patient: intratumoral heterogeneity for PD-L1 was found in $76 \%$ of cases in at least one cell compartment (Supplementary Table 8).

\section{Correlations among variables}

The amounts of TILs and RILs were mutually positively correlated both in the primary tumor $(\mathrm{p}=0.017)$ and in the pulmonary metastases $(\mathrm{p}=0.047)$. In the primary tumors, the amount of $\mathrm{CD} 4^{+}$and $\mathrm{CD} 8^{+}$cells in the immune infiltrate was positively correlated, both in TILs and in RILs (respectively, for TILs: $\mathrm{p}=0.019$, Pearson coefficient $[\rho]=0.46$, moderate correlation, and for RILs: $\mathrm{p}=0.002 ; \rho=0.59$, moderate correlation). In the metastases, this applied to RILs only ( $\mathrm{p}=0.002 ; \rho=0.59$, moderate correlation), but instead the amounts of $\mathrm{CD}^{+}$in TILs and 
Figure 3. Intratumoral heterogeneity. Heterogeneity of expression for PD-L1 at the immunohistochemistry assay in a case of primary renal cell carcinoma (20x) from the study population.

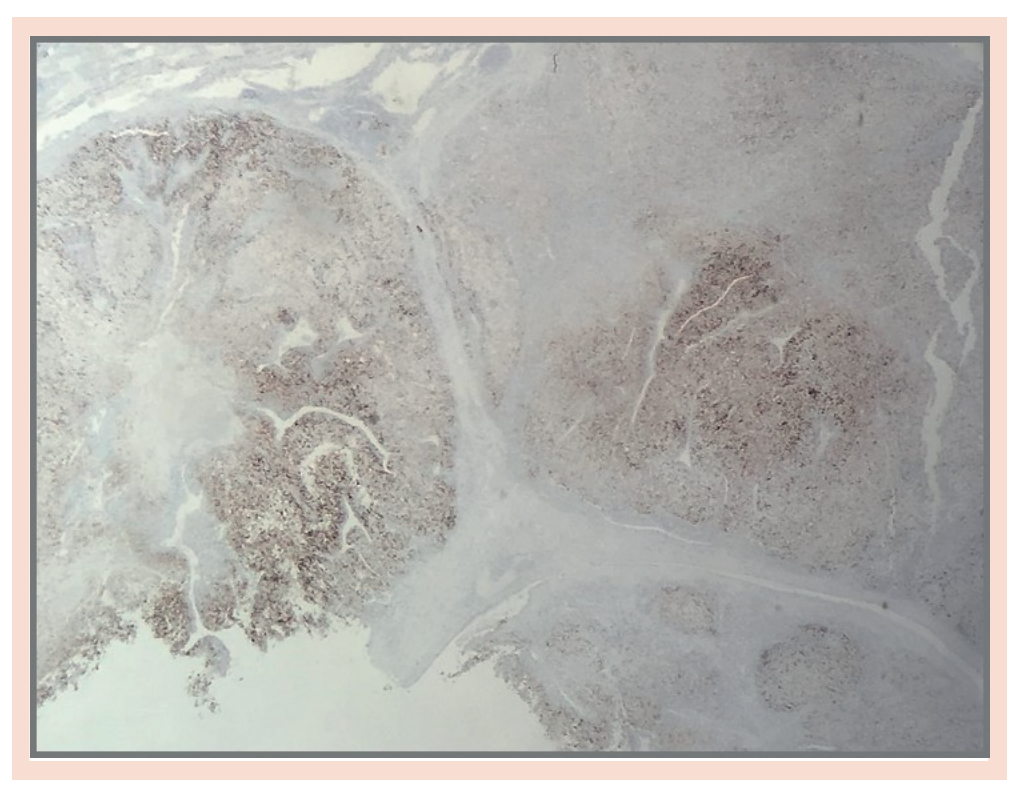

RILs were very strongly positively related ( $\mathrm{p}<0.001 ; \rho=0.93)$; similarly, the amount of CD4 ${ }^{+}$in TILs and RILs were mutually positively related ( $\mathrm{p}=0.028 ; \rho=0.44$, moderate correlation).

In the primary tumor, the amount of $\mathrm{CD}^{+}{ }^{+}$was positively related to the intensity of PD- 1 expression both in TILs and RILs, respectively, with $\mathrm{p}=0.007, \rho=0.52$ and $\mathrm{p}=0.025, \rho=0.045$ (moderate correlations).

The amount of $\mathrm{CD}^{+}$was proportional in TILs and RILs of the primary tumor $(\mathrm{p}<0.001 ; \rho=0.82$, strong correlation); both were, respectively, positively related to those of the metastases and both positively correlated with the intensity of PD-L1 expression in TILs of the primary tumor (respectively, $\mathrm{p}=0.001 ; \rho=0.64$ for CD8 ${ }^{+}$TILs amount and $\mathrm{p}<0.001 ; \rho=0.65$ for $\mathrm{CD}^{+}$RILs amount, moderate correlations; Supplementary Figures 4 \& 5). Moreover, both were also positively related to the intensity of PD-L1 expression in RILs of metastases (respectively, $\mathrm{p}=0.045 ; \rho=0.4$ and $\mathrm{p}=0.04, \rho=0.41$, moderate correlations).

In the metastases, the quantity of $\mathrm{CD}^{+}$TILs resulted to be positively related to the intensity of PD- 1 expression on TILs ( $\mathrm{p}=0.014$ and $\rho=0.49$, moderate correlation) and of PD-L1 expression on tumor cells $(\mathrm{p}=0.012$, $\rho=049$, moderate correlation) and RILs ( $p=0.011, \rho=0.5$, moderate correlation). The amount of CD $8^{+}$RILs was, in turn, positively related to the intensity of PD-1 expression on RILs and TILs (with $\mathrm{p}=0.019, \rho=0.46$ and with $\mathrm{p}=0.047, \rho=0.4$, respectively, moderate correlations) and to the intensity of PD-L1 expression on tumor cells and RILs (with $\mathrm{p}=0.044, \rho=0.41$ and with $\mathrm{p}=0.011, \rho=0.5$, respectively, moderate correlations).

The presence of $\mathrm{CD}^{+}$in TILs and RILs of the metastases was positively related to the age at diagnosis (Supplementary Figure 6), respectively, with $\mathrm{p}=0.004$ (moderate correlation), $\rho=0.56$ and $\mathrm{p}=0.026, \rho=0.44$ (moderate correlation). The TILs intensity score $(0 / 1+$ vs $2++/ 3+++)$ was also related with age, with more probability of higher TILs score for patients over 60 years $(\mathrm{p}=0.046)$.

The Fuhrman grade of the primary tumor was inversely related to the CD4/CD8 ratio of TILs, both in the primary cancer $(\mathrm{p}=0.044, \rho=-0.4$, moderate correlation) and in the metastasis $(\mathrm{p}=0.047, \rho=-0.4$, moderate correlation); indeed, tumors of higher grade had greater amounts of CD8 ${ }^{+}$lymphocytes in the metastases, both in TILs and RILs (respectively, $\mathrm{p}=0.027, \rho=0.4$ and $\mathrm{p}=0.043, \rho=0.4$, moderate correlations), as shown by Supplementary Figure 7.

PD-1 expression on TILs and RILs of the primary tumor was directly correlated, both quantitatively ( $\mathrm{p}<0.001$, $\rho=0.83$; strong correlation) and as categorical variable (odds ratio [OR]: 12.5 [95\% CI: 1.6-97.6], $\mathrm{p}=0.017$ ).

Primary tumors with more necrosis were more likely to express PD-1 in RILs, with a threshold greater than $10 \%(\mathrm{p}=0.012)$. The quantitative expression of PD-1 in TILs and RILs of the metastasis shown a positive mutual correlation ( $\mathrm{p}<0.001, \rho=0.78$; strong correlation); both parameters resulted to be related to PD-L1 expression intensity in the metastasis (with $\mathrm{p}=0.017, \rho=0.47$ and with $\mathrm{p}=0.044, \rho=0.4$, respectively, moderate correlations) and only the first one was also related to PD-L1 expression intensity in TILs of the metastasis $(\mathrm{p}=0.038, \rho=0.42$, moderate correlation). PD-1 and PD-L1 expressions on TILs of the metastases were therefore associated. 


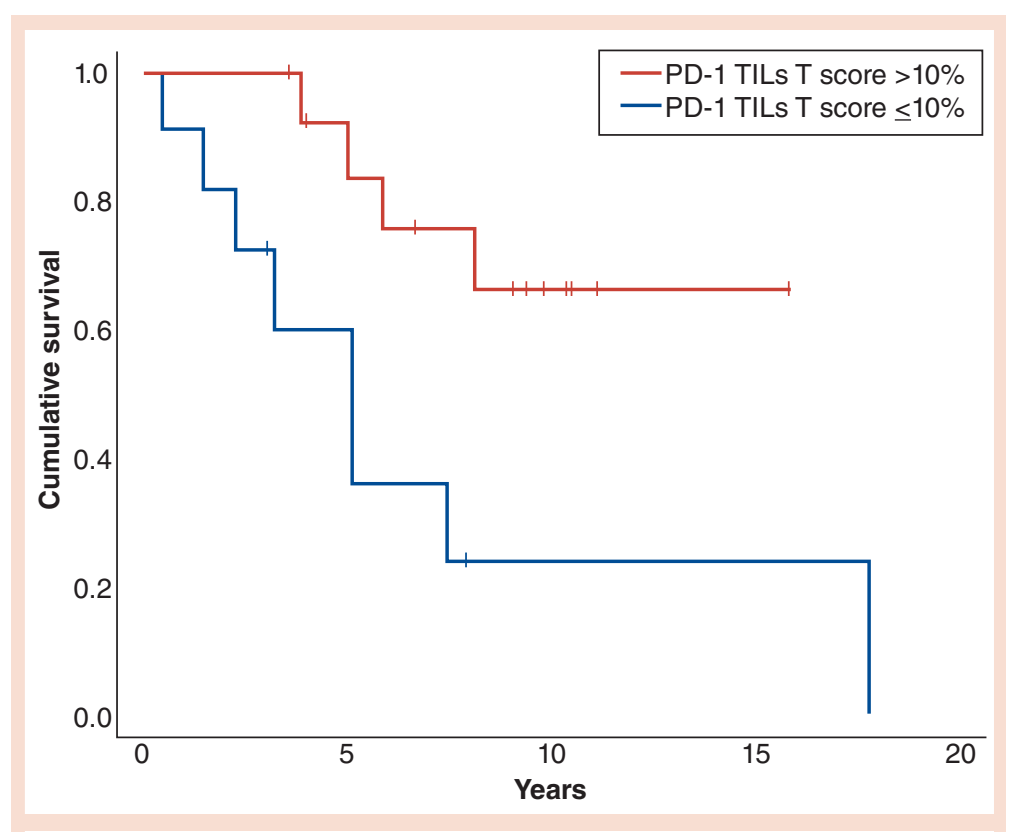

Figure 4. The intensity of PD-1 expression in tumor-infiltrating lymphocytes of the primary tumor (T) was related to better overall survival from nephrectomy. A positive prognostic impact emerged for PD-1 expression greater than $10 \%$ in TILs of the primary tumor. Median overall survival at the median follow-up of 9.8 years was not reached in cases over this threshold versus median overall survival of 5.1 years (95\% Cl: $2.7-7.5$ ) for cases with lower expression levels (mean 12.4 vs 7.3 years); $\mathrm{p}=0.017$. TIL: Tumor-infiltrating lymphocyte.

PD-L1 expression intensity in the primary tumor demonstrated a positive correlation with its own heterogeneity of expression ( $p<0.001$ ), thus the highest expression intensity was found in the most heterogeneous tumors. PD-L1 positivity on TILs and RILs of the primary tumor had a strongly positive mutual correlation, with OR: 52.5 (95\% CI: 4.05-680.95), $\mathrm{p}=0.001$.

PD-L1 expression intensity on TILs of the primary tumor was positively related to the amounts of CD8 ${ }^{+}$in TILs and RILs of metastases (respectively, $\mathrm{p}=0.037, \rho=0.42$ and $\mathrm{p}=0.013, \rho=0.49$, moderate correlations). PD-L1 expression intensity on RILs of the primary tumor, in turn, was significantly related to its own heterogeneity ( $\mathrm{p}<0.001)$ : the highest expression intensity was found in RILs with the most heterogeneous PD-L1 expression. Also in the case of the metastases, the intensity of PD-L1 expression on RILs was significantly related to its heterogeneity $(\mathrm{p}=0.004)$ : the peaks of highest PD-L1 expression intensity were found in the most heterogeneous cases. The intensity of PD-L1 expression on RILs of the metastases also showed a positive correlation with age ( $\mathrm{p}=0.003, \rho=0.57$, moderate correlation).

\section{Survival analysis}

Several immunological variables of the primary tumor correlated with OS from nephrectomy. PD-1 expression greater than $10 \%$ in TILs was associated with improved OS (mOS not reached at the median follow-up of 9.8 years vs $\mathrm{mOS}=5.1$ years [95\% CI: 2.7-7.5]; $\mathrm{p}=0.017$; Figure 4). A trend in favor of a better OS was also observed for higher PD-L1 heterogeneity in tumor cells and TILs (Supplementary Figures 8 \& 9). A higher TILs intensity score showed a trend for worse mOS (5.1 years vs not reached [NR]; Supplementary Figure 10). The OS difference based on PD-L1 positivity on tumor cells was instead not significant.

Among variables analyzed on the pulmonary metastases, those with a potential correlation with OS from nephrectomy were: the presence of necrosis, correlated to a worse OS ( $p=0.031)$; high PD-L1 positivity on TILs and RILs, related to a better OS ( $\mathrm{p}=0.005$ and 0.049 , respectively); PD-L1 heterogeneity on TILs, related to improved OS (NR vs 5.1 years [95\% CI: 4-6.2] for cases with homogeneous PD-L1 expression, $\mathrm{p}=0.032$ ).

The surgical interval was correlated to the Classification of Malignant Tumors (TNM) stage of disease: 35.7 months (95\% CI: 18.5-52.8) stage I; 37.9 months (95\% CI: 0-99.7) stage II; 25.2 months (95\% CI: 15.7-34.7) stage III; 2.7 months (95\% CI: 0-16.7) stage IV, $\mathrm{p}=0.045$. PD-1 expression on TILs of the primary tumor also favorably influenced the surgical interval (37.8 months [95\% CI: 30.7-44.9] for positive cases vs 15.7 for negative cases [95\% CI: 0-42.3], p = 0.032; surgical interval of 37.9 months [95\% CI: 30.4-45.4] for cases $>10 \%$ PD-1 vs 15.7 for cases <10\% PD-1 [95\% CI: 0-36.4], p = 0.006). Finally, the shorter was the surgical interval, the more probably the pulmonary metastases had PD-L1 expression greater than $10 \%$ on TILs, $\mathrm{p}=0.024$. 


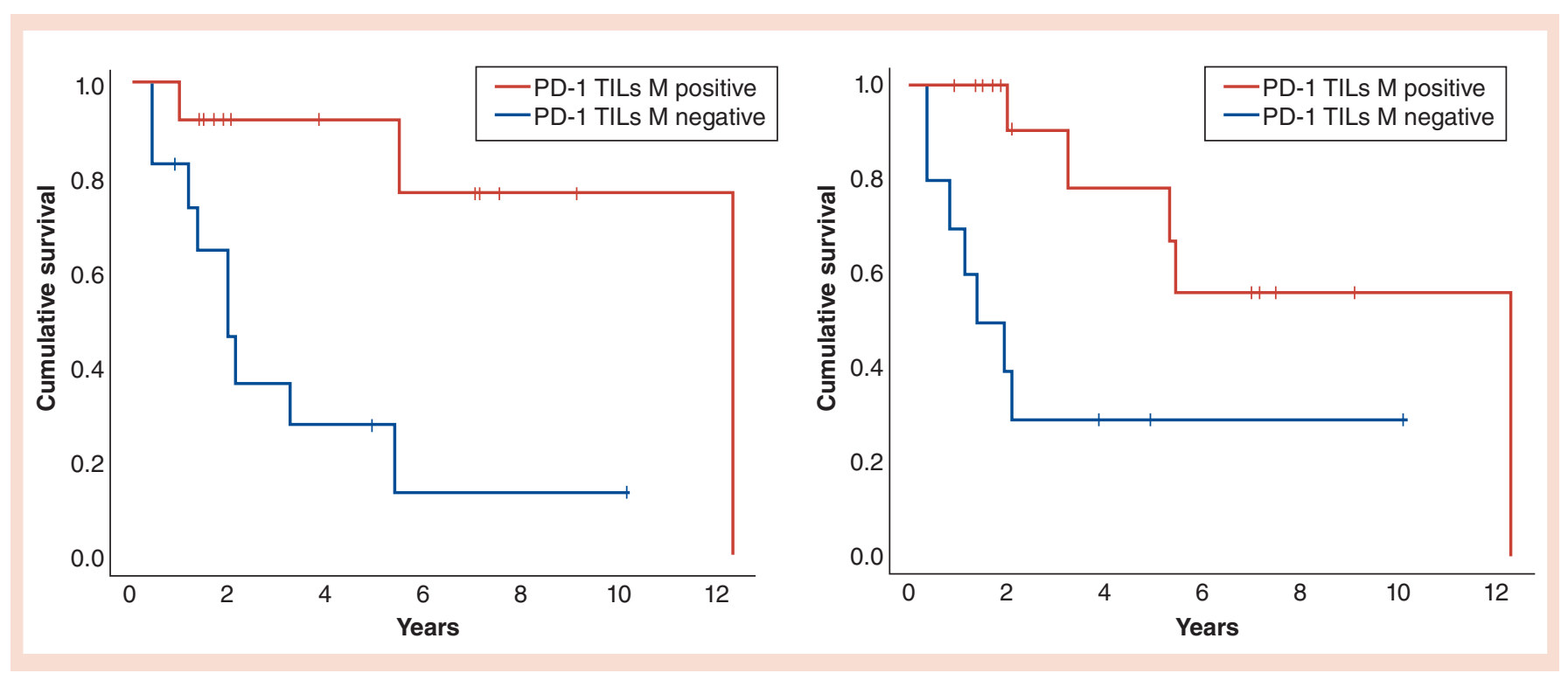

Figure 5. PD-L1 expression on infiltrating lymphocytes of metastases (M) was related to better overall survival from metastasectomy. (A) A positive prognostic impact emerged for PD-L1 positivity in TILs of the metastases. Median overall survival was not reached in PD-L1-positive cases versus median overall survival of 2 years in negative cases (95\% Cl: $1.2-2.7), p=0.003$. (B) A positive prognostic impact emerged for PD-L1 positivity in peritumoral RILs of the metastases. PD-L1 positivity on RILs was related to better overall survival from metastasectomy that was not reached versus 1.4 years for negative cases $(95 \% \mathrm{Cl}: 0.2-2.6), \mathrm{p}=0.012$.

RIL: Renal-infiltrating lymphocyte; TIL: Tumor-infiltrating lymphocyte.

None of the immune variables analyzed on the primary tumors had a significant correlation with OS from metastasectomy. Conversely, high TILs intensity score $(2++/ 3+++)$ in the metastases was related to worse OS $(\mathrm{p}=0.005)$. PD-L1-positive cases on TILs and RILs of metastases had better OS from metastasectomy (respectively, NR vs 2 years [95\% CI: $1.2-2.7], \mathrm{p}=0.003$ and $\mathrm{NR}$ vs 1.4 years [95\% CI: $0.2-2.6], \mathrm{p}=0.012$, respectively; Figure 5); this result was confirmed by the quantitative analysis of PD-L1 on TILs ( $\mathrm{p}=0.038$ ). Higher PD-L1 intralesional heterogeneity on TILs of metastases was also related to improved OS from metastasectomy (NR at the median follow-up vs 2.1 years [95\% CI: 0.4-3.7] for cases with homogeneous PD-L1 expression; $\mathrm{p}=0.024$; Supplementary Figure 11). A similar trend was shown for PD-L1 heterogeneity on RILs (Supplementary Figure 12).

\section{Discussion}

mRCC patients with extracranial radically resected metastases have a survival rate of $45 \%$ at 5 years according to recent reviews [30]. The mOS of our series was 5.5 years with a 5 -year survival of $58 \%$ after metastasectomy. These figures suggest that our patients were positively selected among the general spectrum of mRCC patients, because of favorable criteria such as the oligometastatic disease and the feasibility of a surgical approach. This favorable setting must be taken in account while considering the relevance of the prognostic and predictive elements emerging from our findings that are not necessarily representative of the average renal cancer cases. Thus, every consideration about prognosis and outcomes should be only applied to corresponding clinical contexts.

The immune-context of RCC, well known as a highly immunogenic tumor, is currently poorly understood in the light of the new therapeutic possibilities with immune-checkpoint blockade, and the biological basis of the immune tolerance is still unknown.

Literature data indicate a correlation between PD-1 expression by the tumor immune infiltrate and higher stage, higher grade, more aggressive features (coagulative tumor necrosis, sarcomatoid component) and poorer prognosis of RCC [14]. Conversely, we observed a favorable prognostic impact of PD-1 expression on TILs (Figure 4) both on OS and surgical intervals. This discrepancy can be explained. First, data presented by the literature regarded surgical populations of patients radically resected for early-stage RCC: since our population is composed only by metastatic patients, they have been probably already selected for certain features, among those PD-1 expression may characterize diseases more likely to become advanced. Indeed, in our cases too, more necrotic primary tumors were 
more likely to recall a PD-1-positive peritumoral infiltrate. Nevertheless, in metastatic patients, such biomarker could contrarywise represent a good sign of immune-activation, since PD-1 is notoriously expressed by activated $\mathrm{T}$ cells, as a physiological mechanism of self-regulation. Thus, we could interpret our data hypothesizing that the immune infiltrate of patients expressing PD-1-positive TILs could be more functional than the average, ameliorating the prognosis in presence of metastatic disease. This hypothesis is furtherly supported by our finding of a positive correlation between the expression of PD-1 in the immune context and the amount of cytotoxic $\mathrm{CD}^{+} \mathrm{T}$ cells in the metastases, suggesting a more immunogenic tumor, maybe more likely to represent a good substrate for immune-checkpoint blockade, thus exploiting the 'inflamed' tumor context. This is also suggested by the association of PD-1 expression with PD-L1 expression on both tumor cells and immune cells of the same metastases, in turn evidenced by our results (and confirmed by the literature [14]), despite the still clinical doubtful impact of the true predictive value of this biomarker toward the new immunotherapy in the case of RCC. The immune context and its clinical significance might be different if analyzed in the primary tumor, where PD-1 expression was rather correlated to the quantity of $\mathrm{CD}^{+} \mathrm{T}$ cells. Recent data suggest that tumors with aggressive features, such as necrosis, high grade, sarcomatoid or rhabdoid component, are more likely to be immunogenic, and the same features may be predictive of response to anti-PD-1/PD-L1 therapies [31,32]. Most studies have considered these features only in primary tumors; we investigated these aspects also at secondary sites, showing higher number of $\mathrm{CD}^{+}$lymphocytes in metastases from tumors with higher grade, thus subtending greater immune activation (Supplementary Figure 7).

As already discussed, despite in many solid tumors the inflammatory infiltrate is related to good prognosis [7], the literature about RCC supports an unfavorable prognostic role for TILs and PD-L1 expression on tumor cells $[8-10,13-15]$.

Our results relating TILs in the primary tumor to OS from nephrectomy showed a trend supporting such evidence (Supplementary Figure 10), despite the limit of a small sample size, that not allowed a comparison between negative and positive cases (data about the presence of TILs were dichotomized by comparing score $0 / 1+$ with score $2++/ 3+++$, because of the small number of cases lacking TILs). In metastatic lesions, as explored in this study for the first time, higher grades of inflammatory infiltrate (TILs intensity $2++/ 3+++$ ) had a poor prognostic meaning.

The prognostic impact of PD-L1 expression on tumor cells was instead not significant in our small population. Conversely, about the role of PD-L1 expression in the immune infiltrating cells, we found for the first time that the positivity of such biomarker on TILs and RILs of the metastases configured a positive prognostic value, which was also proportional to the expression intensity (Figure 5). PD-L1 expression on TILs and RILs of the tumor could represent a sign of possible enhanced (or susceptible to be easily enhanced through immune-checkpoint blockade) immune response, as suggested by its proportional relationship with the amount of $\mathrm{CD} 8^{+}$lymphocytes in the peritumoral and intratumoral infiltrate (Supplementary Figures $4 \& 5$ ). In the literature, PD-L1 tumor expression also subtend immune-activation, as demonstrated by its positive relationship with lymphocyte density in tumor microenvironment [32-34]. Interestingly, we found a positive correlation between the intensity of PD-L1 expression on RILs of the metastases and the age of patients; notably, older adults seem to benefit from CKI as well as younger patients, possibly as the result of an enhanced immune response [35]. This hypothesis is also supported by our finding that the presence of $\mathrm{CD}^{+}$in TILs and RILs of the metastases was positively related to the age at diagnosis.

One of the major findings of the present study concerns the heterogeneity of the immune infiltrate both within a given tumor mass and between paired primaries and metastases (Supplementary Tables 2-5). Rates of intralesional heterogeneity for at least one lymphocyte subpopulation $\left(\mathrm{CD}^{+}\right.$or $\mathrm{CD}^{+}$or both) ranged from $52 \%$ in TILs to $76 \%$ in RILs of the primary tumor and from $28 \%$ in TILs to $48 \%$ in RILs of the metastases. Similarly, intralesional heterogeneity for PD-L1 expression raised from $44 \%$ in the primary tumors to $56 \%$ in the metastases. Even more, intratumoral heterogeneity for PD-L1 expression reached $76 \%$ of cases, a rate higher than that evidenced in the literature [26]. Intratumoral heterogeneity can lead to the underestimation of the tumor landscape portrayed from single and outdated tumor-biopsy samples, presenting major challenges to biomarkers development and therapeutic management (and thus becoming responsible of treatment failure). Our findings not only reinforce the need of wide tumor sampling but they also warn against the use of immune variables as assessed in the primary tumor to predict the outcome in the metastatic setting.

The assessment of PD-L1 expression, repeatedly (and sometimes inappropriately) used in clinical trials as a decision-making parameter, was shown to be unreliable in the present analysis. Indeed, it represents a highly heterogeneous element, which clinical meaning still escapes, probably because of its misunderstood dynamism 
and spatiotemporal inconstancy in the tumor and immune context, which determines its difficulty of proper determination and interpretation. In the light of our results, intratumoral and intralesional heterogeneity could contribute to its improper assessment (Supplementary Tables $6 \& 7$ ). The intralesional variability of PD-L1 expression on tumor cells within the primary tumors and the single metastases was around $16-20 \%$ of cases, with heterogeneous expression (both in qualitative and quantitative terms) among different fields of the same tumor mass. This variability was even higher when assessed on the immune cells: the heterogeneity of PD-L1 expression on TILs and RILs ranged from $24 \%$ of cases within the primary tumor to $36 \%$ of cases within the metastasis. There was also a weak correlation of PD-L1 expression between primary and metastatic sites, as already evidenced by the literature with more complex methods than IHC [25]. As to PD-L1 expression in tumor cells, only a minority of cases were completely concordant between paired samples of primary and secondary lesions (Supplementary Table 8). Ultimately, such results, supported by very recent literature data [36], suggest that a single core biopsy might not be sufficient to determine PD-L1 expression.

An unexpected result of our study was the observation that heterogeneity of the immune context showed a positive trend of correlation toward better survival (Supplementary Figures 8, 9, $12 \& 13$ ), a finding that requires to be discussed. Tumors with heterogeneous immune responses might be immunologically more plastic, more prone to respond to external variables and more likely to be molded over time by pharmacological treatments. This interpretation is supported by the observation that PD-L1 heterogeneity decreases the negative prognostic impact of its levels of expression. In fact, we found higher probability of heterogeneity among the long survivors, irrespective of the intensity of expression. Interestingly, in support of such hypotheses, a recent report from a phase III trial demonstrated that different molecular gene expression signatures (GE) correlated with clinical outcomes, prognostic risk groups and tumor histology. The study suggests the idea of a clear distinction between VHL-driven RCC, with high angiogenesis GE, probably more homogeneous and more responsive to targeted therapy, and non-VHL-driven RCC, with T effector GE, more aggressive and heterogeneous features, but more immunogenic and responsive to immunotherapy [27].

This study has major limitations: the retrospective nature, the only explorative purpose, the positive selection of patients and the small sample size distributed over a long-time period, during which the therapeutic options have changed and the expected survival for RCC patients has also modified over time. Despite these clear limitations, which make our prognostic correlations rather weak, the present explorative analysis has the merit to brought out the problem of intratumoral and intralesional heterogeneity of the immune cell context and of the immune-related biomarkers, at a time when such elements are a hot topic of interest and investigations.

\section{Conclusion}

Altogether our study strongly suggests not only that a comprehensive analysis of tumor samples would be mandatory to accurately define the biological and clinical significance of immune biomarkers but it also points out the need to clearly distinguish the meaning of their expression in the different cell compartments. Moreover, time and space variation should be addressed not only within the primary tumor but also between primary and metastases, possibly with a dynamic assessment, repeating the analysis overtime during the course of the disease.

The sequential exposure to different therapeutic agents, with consequent selective pressure, is able to modify the environment of the tumor and therefore its sensitivity to different treatments. A continuous modification of the tumor immune context is conceivable overtime and may be responsible of modulation of tumor aggressiveness and behavior, influencing clinical outcome and responses to therapy.

The awareness of the heterogeneity of RCC, already well known in the field of genomics and proteomics, should be extended to the immune context of this complex disease.

\section{Future perspective}

The continuous modification of the tumor immune context overtime, despite representing a potential trap for effectiveness of systemic treatments, it is far from constituting an inexorable event beyond our control. Contrariwise, the future step in this field goes from the investigation to the potential manipulation, exploiting the knowledge of the immune context to made it susceptible to be modulated for therapeutic purposes.

In this light, such explorative studies will suggest the direction in which to move the search for new druggable targets and checkpoints, which may be different in very similar malignancies or instead they may be the same but take on opposite meaning based on the primary tumor and on the identification site. 
Nevertheless, the immune context manipulation in RCC, far from being involved only in the case of immunotherapy, it could also be exploited in the field of targeted therapy, which if on the one hand it possibly disregards the subjectivity of the individual, on the other hand it relies on molecular pathways unquestionably involved in the pathogenesis of the tumor and in its interaction with the host's immune system.

\section{Summary points}

- The immune context of renal cell carcinoma (RCC) is complex and still scarcely known, although being of crucial interest for its prognostic and probably predictive value.

- RCC heterogeneity has been investigated as a feature of the tumor cells, but it is largely unknown if it concerns also the immune context.

- The present study, with explorative intent, pointed out the immune context heterogeneity in metastatic RCC, confirming its possible prognostic role and expanding its knowledge in the light of the new immunotherapeutic options for this disease.

- The meaning of the diverse lymphocyte subpopulations and of immune checkpoint biomarkers, such as PD-L1 and PD-1, can be very different depending on the way and on the site of assessment, if tumor cells or immune infiltrating cells and, furthermore, if primary or metastatic lesions.

Financial \& competing interests disclosure

The authors have no relevant affiliations or financial involvement with any organization or entity with a financial interest in or financial conflict with the subject matter or materials discussed in the manuscript. This includes employment, consultancies, honoraria, stock ownership or options, expert testimony, grants or patents received or pending, or royalties.

No writing assistance was utilized in the production of this manuscript.

\section{Ethical conduct of research}

The authors state that they have obtained appropriate institutional review board approval (Local Ethical Committee of Parma) and have followed the principles outlined in the Declaration of Helsinki for all human or animal experimental investigations. Informed consent has been obtained from the participants involved where applicable according to the Local Ethical Committee indications.

Author contributions

M Bersanelli, L Gnetti and S Buti designed the study protocol; all the authors contributed to the acquisition and elaboration of data as per their expertise; M Bersanelli, L Gnetti, S Buti, E Varotti and A Cortellini performed the data analysis; M Bersanelli, S Buti and L Gnetti performed the interpretation of data; M Bersanelli and L Gnetti wrote the first draft of the manuscript; all the authors revised it critically for important intellectual content; all the authors provided to the final approval of the version to be published and agreed to be accountable for all aspects of the work in ensuring that questions related to the accuracy or integrity of any part of the work are appropriately investigated and resolved.

Supplementary data

To view the supplementary data that accompany this paper please visit the website at: www.futuremedicine.com/doi/suppl/10.22 17/imt-2018-0097

\section{References}

1. Mennitto A, Grassi P, Ratta R, Verzoni E, Prisciandaro M, Procopio G. Nivolumab in the treatment of advanced renal cell carcinoma: clinical trial evidence and experience. Ther. Adv. Urol. 8(5), 319-326 (2016).

2. Bersanelli M, Buti S. From targeting the tumor to targeting the immune system: transversal challenges in oncology with the inhibition of the PD-1/PD-L1 axis. World J. Clin. Oncol. 8(1), 37-53 (2017).

3. Motzer RJ, Escudier B, McDermott DF et al. Nivolumab versus everolimus in advanced renal-cell carcinoma. N. Eng. J. Med. 373(19), 1803-1813 (2015).

4. Motzer RJ, Tannir NM, McDermott DF et al. Nivolumab plus ipilimumab versus sunitinib in advanced renal-cell carcinoma. N. Engl. J. Med. 378(14), 1277-1290 (2018).

5. Massari F, Santoni M, Ciccarese C et al. PD-1 blockade therapy in renal cell carcinoma: current studies and future promises. Cancer Treat Rev. 41(2), 114-121 (2015).

6. Ueda K, Suekane S, Kurose $\mathrm{H}$ et al. Prognostic value of PD-1 and PD-L1 expression in patients with metastatic clear cell renal cell carcinoma. Urol. Oncol. pii: S1078-1439(18)30251-5. doi: 10.1016/j.urolonc.2018.07.003 (2018) (Epub ahead of print). 
7. Fridman WH, Pagès F, Sautès-Fridman C, Galon J. The immune contexture in human tumours: impact on clinical outcome. Nat. Rev. Cancer 12(4), 298-306 (2012).

8. Kolbeck PC, Kaveggia FF, Johansson SL, Grune MT, Taylor RJ. The relationships among tumor-infiltrating lymphocytes, histopathologic findings, and long-term clinical follow-up in renal cell carcinoma. Mod. Pathol. 5, 420-425 (1992).

9. Bromwich EJ, McArdle PA, Canna K et al. The relationship between T-lymphocyte infiltration, stage, tumour grade and survival in patients undergoing curative surgery for renal cell cancer. Br. J. Cancer. 89, 1906-1908 (2003).

10. Nakano O, Sato M, Naito Y et al. Proliferative activity of intratumoral CD8(+) T lymphocytes as a prognostic factor in human renal cell carcinoma: clinicopathologic demonstration of antitumor immunity. Cancer Res. 61, 5132-5136 (2001).

11. Jensen HK, Donskov F, Nordsmark M, Marcussen N, von der Maase H. Increased intratumoral FOXP3-positive regulatory immune cells during interleukin-2 treatment in metastatic renal cell carcinoma. Clin. Cancer Res. 15(3), 1052-1058 (2009).

12. Giraldo NA, Becht E, Vano Y et al. Tumor-infiltrating and peripheral blood T cell immunophenotypes predict early relapse in localized clear cell renal cell carcinoma. Clin. Cancer Res. 23(15), 4416-4428 (2017).

13. Giraldo NA, Becht E, Pagès F et al. Orchestration and prognostic significance of immune checkpoints in the microenvironment of primary and metastatic renal cell cancer. Clin. Cancer Res. 21(13), 3031-3040 (2015).

14. Thompson RH, Dong H, Lohse CM et al. PD-1 is expressed by tumor-infiltrating immune cells and is associated with poor outcome for patients with renal cell carcinoma. Clin. Cancer Res. 13, 1757-1761 (2007).

15. Kang MJ, Kim KM, Bae JS et al. Tumor infiltrating PD-1-positive lymphocytes and FoxP3-positive regulatory T cells predict distant metastatic relapse and survival of clear cell renal cell carcinoma. Transl. Oncol. 6, 282-289 (2013).

16. Francisco LM, Salinas VH, Brown KE et al. PD-L1 regulates the development, maintenance, and function of induced regulatory $\mathrm{T}$ cells. J. Exp. Med. 20613, 3015-3029 (2009).

17. Mc Dermott DF, Drake CG, Sznol M et al. Survival, durable response, and long-term safety in patients with previously treated advanced renal cell carcinoma receiving nivolumab. J. Clin. Oncol. 33(18), 2013-2020 (2015).

18. Motzer RJ, Rini BI, McDermott DF et al. Nivolumab for metastatic renal cell carcinoma: results of a randomized Phase II trial. J. Clin. Oncol. 33, 1430-1437 (2015).

19. McDermott DF, Sosman JA, Sznol M et al. Atezolizumab, an anti-programmed death-ligand 1 antibody, in metastatic renal cell carcinoma: long-term safety, clinical activity, and immune correlates from a Phase Ia study. J. Clin. Oncol. 34(8), 833-842 (2016).

20. Herbst RS, Soria JC, Kowanetz M et al. Predictive correlates of response to the anti-PD-L1 antibody MPDL3280A in cancer patients. Nature 515, 563-567 (2014).

21. Choueiri TK, Figueroa DJ, Fay AP et al. Correlation of PD-L1 tumor expression and treatment outcomes in patients with renal cell carcinoma receiving sunitinib or pazopanib: results from COMPARZ, a randomized controlled trial. Clin. Cancer Res. 21, 1071-1077 (2015).

22. Gerlinger M, Rowan AJ, Horswell S et al. Intratumor heterogeneity and branched evolution revealed by multiregion sequencing. $N$. Engl. J. Med. 366(10), 883-892 (2012).

23. Gerlinger M, Horswell S, Larkin J et al. Genomic architecture and evolution of clear cell renal cell carcinomas defined by multiregion sequencing. Nat Genet. 46(3), 225-233 (2014).

24. Crusz SM, Tang YZ, Sarker SJ et al. Heterogeneous response and progression patterns reveal phenotypic heterogeneity of tyrosine kinase inhibitor response in metastatic renal cell carcinoma. BMC Med. 14(1), 185 (2016).

25. Jilaveanu LB, Shuch B, Zito CR et al. PD-L1 expression in clear cell renal cell carcinoma: an analysis of nephrectomy and sites of metastases. J. Cancer 5(3), 166-172 (2014).

26. Callea M, Albiges L, Gupta M et al. Differential expression of PD-L1 between primary and metastatic sites in clear-cell renal cell carcinoma. Cancer Immunol. Res. 3(10), 1158-1164 (2015).

27. Rini BI, Huseni M, Atkins MB et al. Molecular correlates differentiate response to atezolizumab (atezo) + bevacizumab (bev) vs sunitinib (sun): results from a Phase III study (IMmotion151) in untreated metastatic renal cell carcinoma (mRCC). Abstract LBA31. Presented at: ESMO Annual Meeting, Genitourinary Cancers / Cancer Immunology and Immunotherapy /Anticancer agents \& Biologic Therapy, Munich, Germany, 20 October 2018.

28. Everitt BS. The Cambridge Dictionary of Statistics. Cambridge University Press, Cambridge, New York, UK, ISBN 0521593468 (1998).

29. Reed GF, Lynn F, Meade BD. Use of coefficient of variation in assessing variability of quantitative assays. Clin. Diagn. Lab. Immunol. 9(6), 1235-1239 (2002).

30. Loh J, Davis ID, Martin JM, Siva S. Extracranial oligometastatic renal cell carcinoma: current management and future directions. Future Oncol. 10(5), 761-774 (2014).

31. Joseph RW, Millis SZ, Carballido EM et al. PD-1 and PD-L1 expression in renal cell carcinoma with sarcomatoid differentiation. Cancer Immunol. Res. 3(12), 1303-1307 (2015).

32. Vieira T, Antoine M, Hamard C et al. Sarcomatoid lung carcinomas show high levels of programmed death ligand-1 (PD-L1) and strong immune-cell infiltration by TCD3 cells and macrophages. Lung Cancer 98, 51-58 (2016). 
33. Joseph RW, Parasramka M, Eckel-Passow JE et al. Inverse association between programmed death ligand 1 and genes in the VEGF pathway in primary clear cell renal cell carcinoma. Cancer Immunol. Res. 1, 378-385 (2016).

34. Tatli Dogan H, Kiran M, Bilgin B et al. Prognostic significance of the programmed death ligand 1 expression in clear cell renal cell carcinoma and correlation with the tumor microenvironment and hypoxia-inducible factor expression. Diagn. Pathol. 13(1), 60 (2018).

35. Elias R, Giobbie-Hurder A, Jackson McCleary N, Ott P, Hodi FS, Rahma O. Efficacy of PD-1 \& PD-L1 inhibitors in older adults: a meta-analysis. J. Immunother. Cancer 6, 26 (2018).

36. López JI, Pulido R, Cortés JM, Angulo JC, Lawrie CH. Potential impact of PD-L1 (SP-142) immunohistochemical heterogeneity in clear cell renal cell carcinoma immunotherapy. Pathol. Res. Pract. 214(8), 1110-1114 (2018). 